Please cite as:

R. Seco-Gudiña et al., Eur. Phys. J. Special Topics 224, 1189 (2017)

The final publication is available at link.springer.com

\title{
Dynamics of water condensation over arrays of hydrophilic patches
}

\author{
R. Seco-Gudiña ${ }^{1}$, J. Guadarrama-Cetina ${ }^{2}$, and W. González-Viñas ${ }^{3,4, a}$ \\ 1 Universidad de Navarra, Facultad de Ciencias, Pamplona, Spain \\ 2 Instituto de Alta Investigación, Universidad de Tarapacá, Calle Antofagasta 1520, Casilla \\ 7D, Arica, Chile \\ 3 Universidad de Navarra, Complex Systems Group, Pamplona, Spain \\ 4 Universidad de Navarra, PHYSMED Group, Pamplona, Spain
}

\begin{abstract}
We report experimental results of drop-wise condensation on a wettability patterned substrate. It consists of a 2-d array of hydrophilic patches/spots on a macroscopically hydrophobic surface. We show that in this kind of system, there is not a relevant humidity sink, but the scale and the closeness of the different patches/spots affect the mechanisms which are important during the experiment. These results may provide clues to obtain higher dew yields in arid or semi-arid regions as a way to obtain potable water.
\end{abstract}

\section{Introduction}

Heterogeneous / drop-wise condensation patterns, also known as Breath Figures $(\mathrm{BF})$, are ubiquitous in nature and in everyday life, and have been observed by the scientific community since long time ago [1-3]. Models that describe first quantitative experiments appeared in the late twentieth century after the work of Beysens and Knobler [4]. Most of the models are partially coming from first principles and partially from phenomenological theories. Nevertheless, there are a number of different models based on different assumptions, that give rise to the same qualitative results (e.g. kind of power laws in some variable) and are quantitatively consistent, with the already existing experiments.

The basic experimental outcomes brought by BFs are the growth laws and the surface coverage (or occupation factor). The growth law is usually ilustrated by $r(t)$, where $r$ is related to the one-dimensional size of the droplet(s) (i.e. radius) and $t$ is time, while the surface coverage $\epsilon^{2}$ is the area of the projection of the drops on the substrate divided by the total substrate area. The BF behavior changes according to different regimes, that in the more basic set-up (hydrophobic homogeneous substrate where drop-wise condensation occurs) are four, that is to say: $i$ ) the nucleation regime is related to a thermally activated process ([5], and references therein). After drops have nucleated, ii) there is an independent droplet growth regime, where drops barely interact with each other. This latter stage is the most studied till now. After some time, iii) the drops interact highly non-linearly by coalescing. This stage leads, at

a e-mail: wens@unav.es 
long times, to a self-similar growth and a constant surface coverage. At the end, this constant surface coverage may vary, because of $i v$ ) the appearance of new nuclei families.

As an example, in the second regime, the growth of an isolated drop [6,7] follows a different growth law (assymptotically $r \sim t^{1 / 2}$, where $r$ is the radius of the drop) than a dense pattern of drops (assymptotically $r \sim t^{1 / 3}$, where $r$ is the average radius of droplets) do [8]. Usually, the growth of an isolated droplet does not occur, except when there is a sufficiently large region of inhibited condensation (RIC) surrounding a condensed droplet. This could happen with a salty drop, which is highly hygroscopic and acts as a humidity sink. As the concentration of salt in the drop diminishes as it absorbs more water, the growth law is not $r \sim t^{1 / 2}$, but $r \sim t^{1 / 5}$ (assymptotically) [9]. In this latter case, the surrounding breath figure is separated from the salty drop by a RIC as, there, the humidity is depleted. On the other hand, this breath figure grows following the well known power law with $1 / 3$ exponent, depending on the relative distance from the drop [9]. There are other known humidity sinks which are relevant to understand heterogeneous condensation and its applications [10,11]. As a matter of fact, the growth laws depend on many experimental parameters [12-14] like the surface tension, the supersaturation, the dimensionality of the surface, the different vapors in the atmosphere, etc. It can be shown that if the exponent without appreciable coalescence is $\alpha$, in the self-similar regime it becomes $3 \alpha$ (for 3 - $\mathrm{d}$ drops on 2-d substrates $[15,16,5])$.

Breath figures are important in several applications, as they are e.g. in harvesting of drinking water [17], in the increase of efficiency in energy transfer [18,19], to perform soft-patterning [20], to promote/avoid condensation of dew or deposition of frost [2123 , in understanding the effect of humid air in ecological adaptation of some species [24], among many others.

Here, we aim at studying the effect of hydrophilic patches on the surrounding BF. Specifically we are interested in the main differences to the humidity sinks of previous research [9] (depicted above), by looking at the typical outcomes of BFs (surface coverage and growth laws) and the possible existence of RIC. Although most of the hygroscopic surfaces are also hydrophilic, the converse is not necessarily true. As a matter of fact, a hydrophilic surface, once slightly wet is equivalent to a growing droplet with a well-defined boundary. In this case the projection of the droplet to the surface, at short times, does not change, but its volume does. While a hygroscopic surface like a salt crystal (or salty droplet when it becomes wet), makes that the projection of the droplet to the substrate grows indefinitely, but its hygroscopic power reduces as water is absorbed. Another relevant difference in which we are going to focus is the region of inhibited condensation. We studied the effect of an isolated lecithin patch and, as well, of a 2-d array of hydrophilic patches on the BF. We show that, although macroscopically separated hydrophilic patches do not affect the behavior of BF, when they are microscopically separated they do.

\section{Experimental set-up}

The condensation pattern $(\mathrm{BF})$ is induced on a substrate kept at $(10 \pm 0.5){ }^{\circ} \mathrm{C}$, where a constant flow of dry filtered air with saturated water vapor is streamed from two nozzles $\left(T_{\text {room }}=(23 \pm 0.5){ }^{\circ} \mathrm{C}, Q=140 \mathrm{~mL} / \mathrm{min}\right)$. The substrate is inside a Delrin ${ }^{\circledR}$ condensation chamber (cylinder with interior diameter of $10 \mathrm{~cm}$ and height of $1 \mathrm{~cm}$ ), and the substrate is cooled by an electronically controlled Peltier cell. The upper side of the chamber is transparent and allows capture of sequential images with a reflection microscope attached to a CMOS camera, or with a simple observation system (CMOS camera, telescopic lens objective, beam splitter and illumination system) depending 
on the relevant scale to observe. We monitor the temperature by two thermocouples. For more details about the procedure and the set-up, see [25].

The captured images were digitally analyzed by applying OpenCV/C++ routines, which include filtering, thresholding, counting objects and measuring their geometrical features. In the analysis, we exclude the lecithin patches to have only the dynamic description of the BF.

As substrates, we used standard glass microscope slides, which were coated with a suspension of octadecyltrichlorosilane (OTS) in hexamethyldisiloxane (HMDSO) at $1 \%(\mathrm{v} / \mathrm{v})$ by immersion dip-coating. After drying and soft-baking, this coating provides the substrate with both hydrophobic and alcohol-phobic properties which allow to easily deposit patches of lecithin (thickness smaller than $10 \mu \mathrm{m}$ ) on the substrate. We perform three types of experiments, the first one with one lecithin patch, which has a diameter of $(0.42 \pm 0.04) \mathrm{mm}$, on top of the previously obtained hydrophobic substrate. From now onwards we call this type of substrates type 1L. The second one (type 0L) is without lecithin, but with OTS/HMDSO coating and the third one (type $\mathrm{nL}$ ) is with a 2-d square array of lecithin patches (fig. 1) which have an average distance between them of $(3.6 \pm 0.2) \mathrm{mm}$. Their average diameter is $(0.79 \pm 0.06) \mathrm{mm}$.

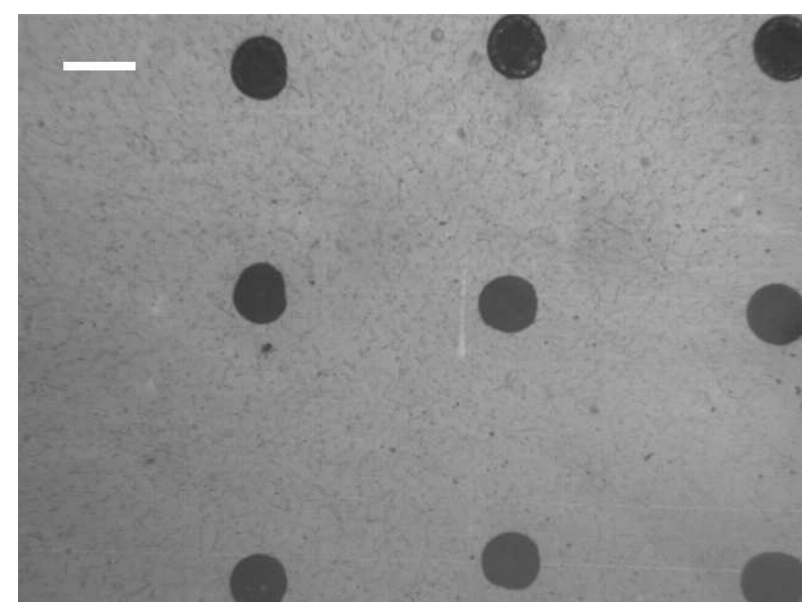

Fig. 1. Substrate (type $\mathrm{nL}$ ) seen from above prior commencing the corresponding experiment. Scale bar is $1 \mathrm{~mm}$.

Lecithin patches become very hydrophilic (fig. 2b), while the bare coated substrate is macroscopically hydrophobic (fig. 2a). The advancing and receding contact angles are measured by the sessile drop method. On lecithin they are $\theta_{A}=(7 \pm 5)^{\circ}$ and $\theta_{R}=(3 \pm 5)^{\circ}$, respectively. On the bare coated glass are $\theta_{A}=(104 \pm 2)^{\circ}$ and $\theta_{R}=(86 \pm 2)^{\circ}$, respectively.

\section{Results and discussion}

Firstly, we report the growth of the condensation pattern on a type 1L substrate. From the very beginning of the experiment there is condensation, and as soon as the heterogeneous condensation can be distinguished, the coalescence regime has started (fig. 3a,d, and supplementary information, movie 1; [26]). In the images of the experiment, a BF appears homogeneously and isotropically distributed around the lecithin 


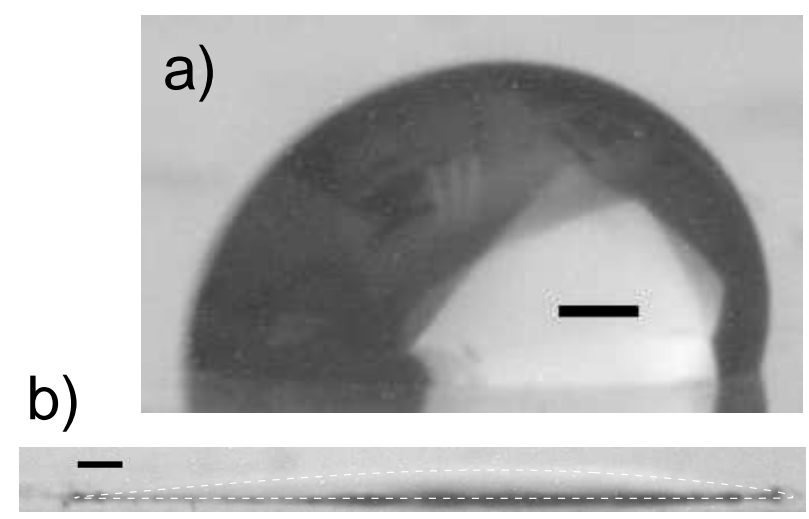

Fig. 2. Drops on the substrate that show advancing and receding stationary contact angles: a) on the coated substrate, and b) on the lecithin patch. Dashed line outlines the shape of the drop, for clarity. Scale bars are $0.5 \mathrm{~mm}$.

patch (which boundary is the overlaid white closed line in the center of figures 3a-c) without a visible RIC. Consequently, the BF does not depend on the lecithin patch. From figure $3 \mathrm{e}$ to figure $3 \mathrm{f}$, we can see two droplets that coalescence. Nevertheless, these two droplets are the product of previous coalescence events (see figure $3 \mathrm{~d}$ ).

We measured the average radius of drops that grow on type $1 \mathrm{~L}$ substrate (fig. $3 \mathrm{~g}$ ). The growth is consistent with an exponent 1 power law (dashed line) which corresponds to a classical coalescence self-similar regime (see section 1 and [5]). We also measured the surface coverage, which is measured as approximately constant, $\epsilon^{2}=0.55 \pm 0.02$.

To explore earlier times than those appearing in fig. 3 where intense coalescence already occurs, we performed the same experiment without lecithin (type 0L substrate) and a higher magnification. In figure 4a-e a sequence of cropped (for clarity) images of the experiment can be seen and a movie from the full images can be found in supplementary information (movie $2 ;[27]$ ).

From the hydrophobic character of the type 0L substrate (fig. 2a) and the relatively low supersaturation, the expected behavior of the system was a low surface coverage at short times as it occurs in 'classical' BF. As an example, an experiment at the same experimental conditions and taken at the same time than figure $4 \mathrm{a}$, but with an Indium Tin Oxide (ITO) coated glass is shown in fig. 4g. Note that the stationary contact angles of water on this substrate are smaller $\left(\theta_{A}=(93 \pm 2)^{\circ}\right.$ and $\theta_{R}=(57 \pm 2)^{\circ}$, [9]) than in our experiments, so the coalescence events should take place even earlier, which clearly does not happen.

The surface coverage from $400 \mathrm{~s}$ to $1200 \mathrm{~s}$ of the (uncropped) images is $\epsilon^{2}=$ $0.55 \pm 0.02$, consistent again with a self-similar regime. Nevertheless, only for times higher than about $750 \mathrm{~s}$, the average radii of drops from the BF correspond to those of a coalescence regime (fig. 4f). The exponent in that region appears to be 3 times the exponent in previous times $(<700 \mathrm{~s})$. However, the latter region is not absent of coalescence and highest surface coverage (see supplementary information, movie 2; [27]). This could be explained as coalescence, when drops are very small, akin to the growth of droplets by addition of monomers which diffuse on the substrate [8]. This issue will require further research.

Bright stains that can be seen in fig. 4a-e (outlined in fig. 4b), may correspond to different phases [28] of the surface coating (OTS in HMDSO) as micellar, inverted, lamellar, ..., or patches [29]. We also performed a higher magnification micrograph of the type 0L substrate before starting the experiment (fig. 5). There, it is possible 

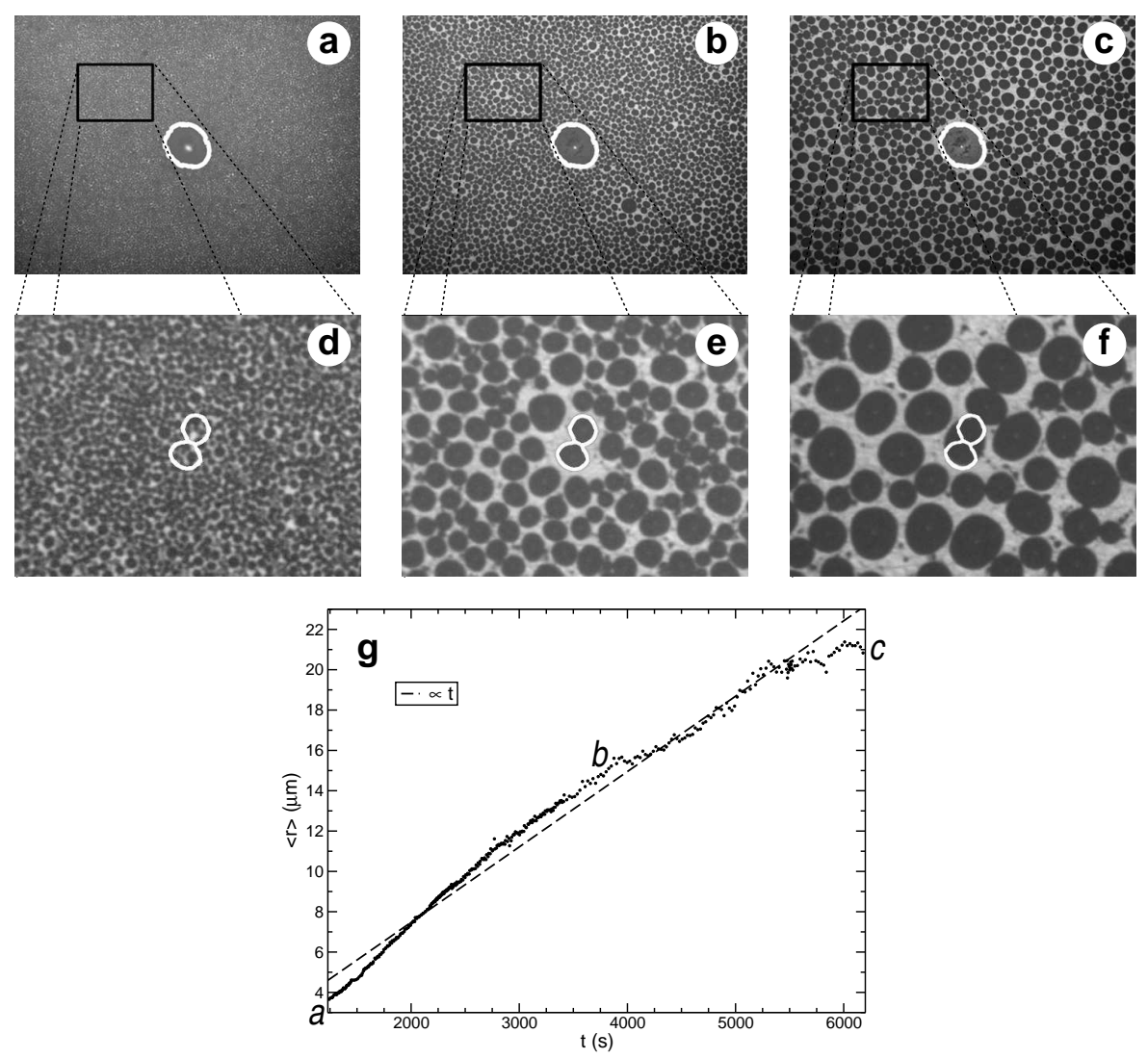

Fig. 3. (a-f) Micrographies of the process of condensation at different times after the beginning of the experiment (1243 s for a and d; $3711 \mathrm{~s}$ for $\mathrm{b}$ and e; $6179 \mathrm{~s}$ for $\mathrm{c}$ and $\mathrm{f}$ ) and different scale $(\mathrm{HFOV}=3.31 \mathrm{~mm}$ for $\mathrm{a}, \mathrm{b}$ and $\mathrm{c}$; HFOV $=0.71 \mathrm{~mm}$ for $\mathrm{d}$, e and $\mathrm{f}$ ). Specifically, $\mathrm{d}$, e and $\mathrm{f}$ are the black rectangles in $\mathrm{a}$, b and c, respectively zoomed. Superimposed white closed lines in a, b and c correspond to the boundary of the initial lecithin patch. Superimposed white closed lines in $\mathrm{d}$, e and $\mathrm{f}$ correspond to the shape of two droplets existing around $3700 \mathrm{~s}$. (g) Average radius of drops corresponding to the experiment with type $1 \mathrm{~L}$ substrate. Dashed line is a proportionally relation fit to the experimental points. Data points corresponding to (a-c) are marked in the plot by slanted font.

to see the observed stains in fig. 4a-e. Moreover, many dark spots are observed that can be assumed as highly hydrophilic, as they are the nuclei of condensation in the experiment.

Finally, the experiment with the type $\mathrm{nL}$ substrate (fig. 1) has been performed. To illustrate what can be seen, we show in fig. 6 , snapshots at short times after the drops are distinguishable, and at long times. Moreover, in supplementary information a movie of the process is presented (movie $3 ;[30]$ ).

In the experiment with a type $\mathrm{nL}$ substrate (fig. 6, left), we can see that the BF is very similar to that on the type $1 \mathrm{~L}$ substrate (fig. $3 \mathrm{~d}$ ), and that the coalescence regime has also started before the drops can be distinguished. In fig. 6 , right, we can see new families of condensed drops at not so long times, which can be explained by the hydrophilic spots on the substrate (fig. 5). There, water is easily adhered and remains after coalescence of drops, even on a macroscopically hydrophobic substrate [31]. This makes possible to observe early presence of new families of droplets as 

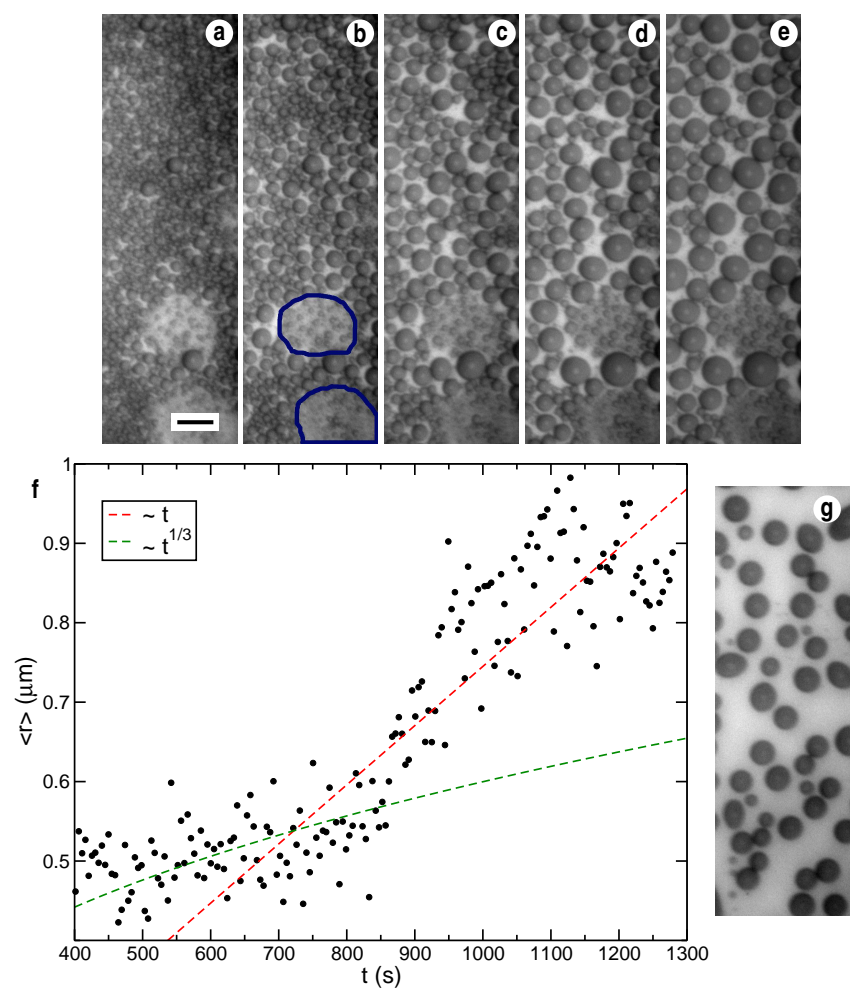

Fig. 4. (a-e) Cropped images from the experiment without lecithin. Time ranges from $400 \mathrm{~s}$ (a) to $1200 \mathrm{~s}$ (e) at regular intervals of $200 \mathrm{~s}$. Scale bar is $20 \mu \mathrm{m}$. In (b), we outline the stains by (blue) solid lines. (f) Average radius of drops of full images corresponding to the experiment. Dashed lines are guides to the eye corresponding to power laws. (g) For comparison with (a), experiment at the same conditions, except that the substrate is Indium Tin Oxide (ITO) coated glass. The time and the scale also is the same.

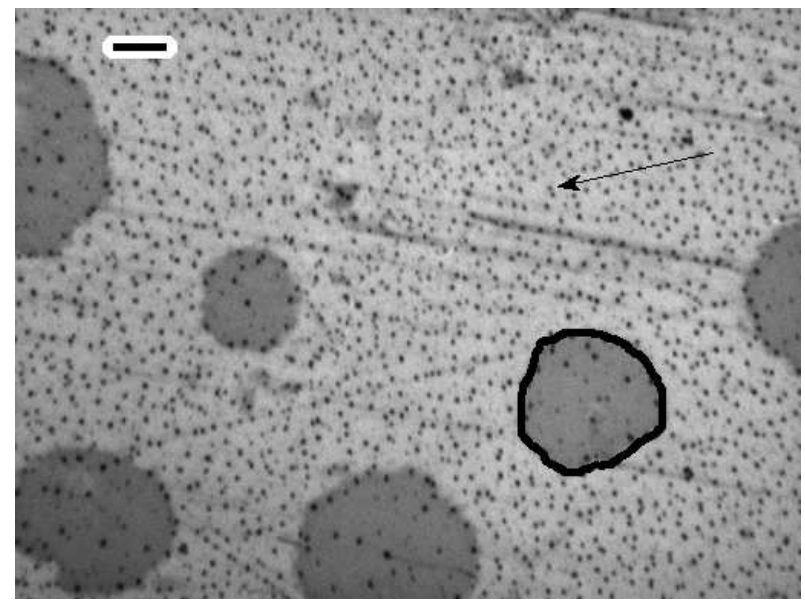

Fig. 5. Higher magnification of type $0 \mathrm{~L}$ substrate prior starting the experiment. There it is possible to observe stains (one of them outlined by a closed solid line) and dark spots (one of the many pointed by an arrow). Scale bar is $9 \mu \mathrm{m}$. 

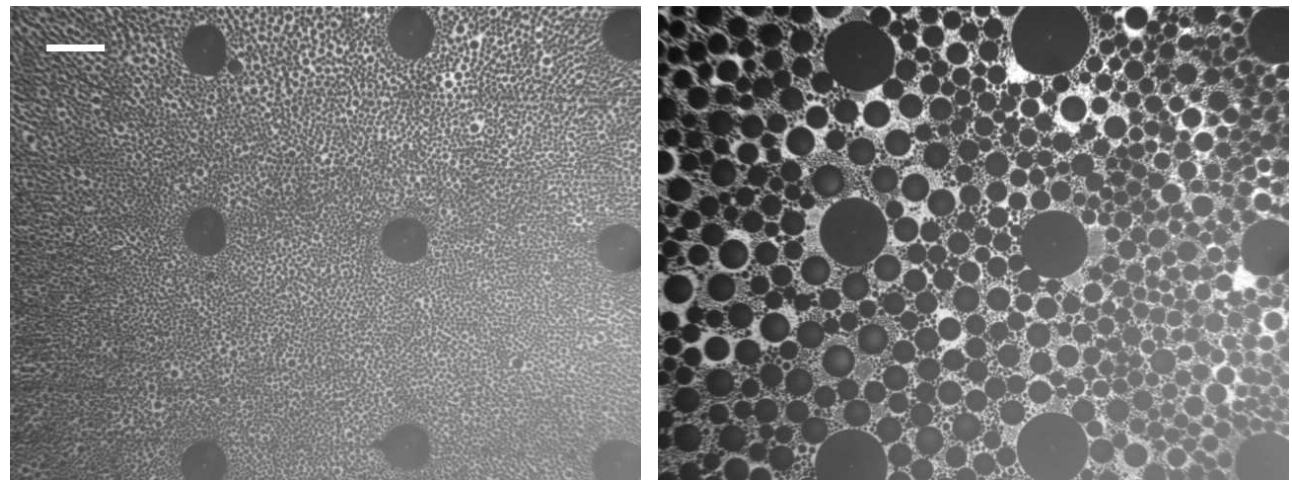

Fig. 6. Snapshots of the experiment on type nL substrate at $6429 \mathrm{~s}$ (left) and $13765 \mathrm{~s}$ (right) after the beginning of the experiment. Scale bar is $1 \mathrm{~mm}$.

coming water molecules do not have to overcome the energy barrier of the nucleation stage, and drops start to grow on the water adhered on the dark spots.

We measured the surface coverage in the time interval with distinguishable drops as $\epsilon^{2}=0.56 \pm 0.02$. In fig. 7 , the growth behavior of the drops on the substrate and on the lecithin patches is shown.
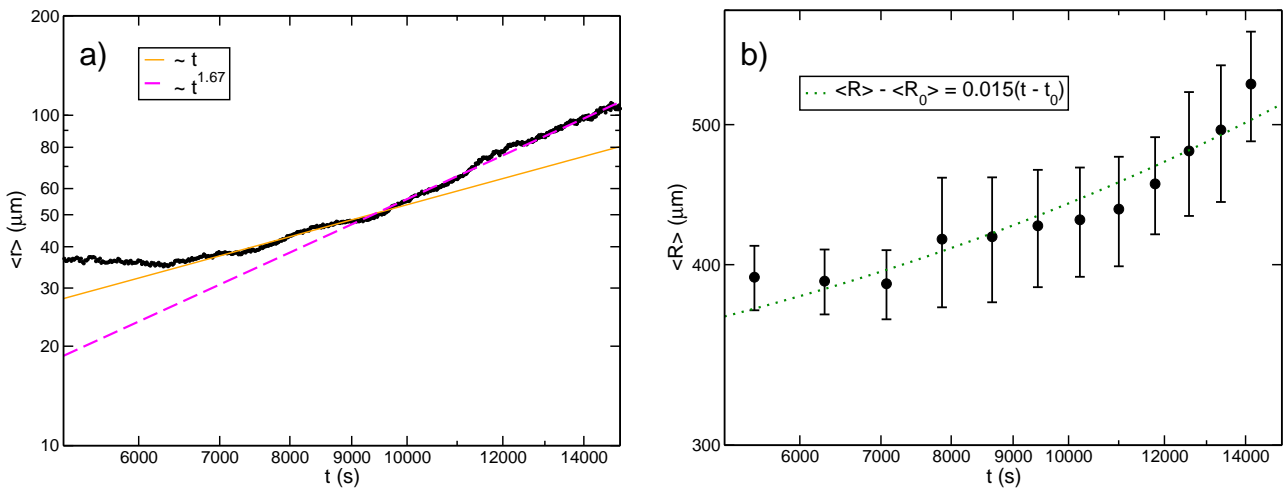

Fig. 7. Experiment with a type $\mathrm{nL}$ substrate. a) Growth law for the average radii of drops not condensed on lecithin (small circles) and its power law fits in the relevant regions (solid and dashed line). b) Growth law for the average radii of the drops grown on the lecithin patches (big circles) and its corresponding linear fit (dotted line).

For small times shown in figure $7 \mathrm{a}$, the size of the drops condensed outside lecithin (fig. 7a, small circles) seems to be constant. This is an artifact because, at the resolution of these images (e.g. as in fig. 6), we cannot measure reliably droplets smaller than around $35 \mu \mathrm{m}$. After that region we can see that the growth is according to the coalescence regime, while at longer times the growth law is with an exponent 1.67. We also plot (fig. 7a, circles) the average radius as seen from above (like eg. in fig. 6) of the drops which grow on the lecithin patches. We can linearly fit the obtained data (dotted line). These drops very early cover the whole lecithin patches due to their high hydrophilicity. Then, drop growth occurs at constant base size, by increasing monotonically the contact angle until its value exceeds the advancing contact angle on the OTS/HMDSO coating (hydrophobic). Thus, the apparent change (i.e. as seen 
from above) of the drop radius does not occur from the beginning and the linear fit is consistent with a displaced power law with exponent 1 .

\section{Concluding remarks}

A wettability pattern at a macroscopic scale on a substrate does not affect appreciably the growth evolution of drops on the hydrophobic region. Also, the RIC around the hydrophilic regions couldn't be observed in our experimental conditions. These constitute notable differences to the $\mathrm{BF}$ growth around a hygroscopic regions (recall type $1 \mathrm{~L}$ and $\mathrm{nL}$ substrates experiments compared to $[9,32])$. However, if the characteristic scale of the hydrophilic patches is small, as well as the average distance between patches is (eg. like in fig. 5), the free (or independent) growth regime is nonexistent or very small, and coalescence starts soon after nucleation (recall fig. 4a compared to fig. 4g). Also, there appear premature new families of droplets on the hydrophilic spots (fig. 6, right). This makes the measured surface coverage in all the regimes studied about the 0.55 , which corresponds to a self-similar regime with a macroscopic contact angle of around $90^{\circ}$ (see the phenomenological relation [33] $\epsilon^{2}=1-\frac{\theta}{200}$, where $\theta$ is the static contact angle expressed in degrees). The exponents of the (power) growth laws are consistent with this regime, except at very short times and small scales (fig. 4f, for times smaller than $700 \mathrm{~s}$ ) and at long times (fig. 7a, for times bigger than $9000 \mathrm{~s}$ ).

We think that the behavior for the growth laws at short times (fig. 4f, for times smaller than $700 \mathrm{~s}$ ) is related to an on-substrate effective diffusion of the "monomers" or small droplets [8] which leads a $1 / 3$ exponent even with coalescence. At very long times (fig. 7a, for times bigger than $9000 \mathrm{~s}$ ) the exponent (1.67) is bigger than expected $(3 \alpha=1)$, and this could be also due to an additional growth of big drops by diffusion on the substrate. Nevertheless, further research is required to confirm the physical mechanism that underlies this result.

Macroscopic hydrophobic character of substrates (e.g. type 0L; and, 1L and nL outside the lecithin patches) can coexist with an early coalescence regime and high surface coverage, if there is a highly dense distribution of nucleation sites/hydrophilic spots. This is of practical interest to increase dew yield in arid regions and to recover water from atmospheric humidity. Also, it allows to maximize energy transfer in the heterogeneous condensation process.

In summary, the three kind of substrates (0L, $1 \mathrm{~L}$ and $\mathrm{nL}$ ) allow us to explore different time and length scales to obtain that i) RIC cannot be observed near hydrophilic regions which are not hygroscopic and, hence, do not act as humidity sinks, ii) macroscopic hydrophilic patches do not appreciably affect the BF in hydrophobic surrounding regions, iii) even a macroscopically hydrophobic substrate with a dense distribution of microscopic hydrophilic spots permit to reach early coalescence regimes with high values of surface coverage, iv) our substrates (type 0L, and not lecithin coated regions of $1 \mathrm{~L}$ and $\mathrm{nL}$ ) favor the adhesion of water to the microscopic spots avoiding the need of a nucleation stage when new families of droplets appear.

In the future, experiments will be performed changing the relative composition of the coating in order to vary the density and the typical scale of the hydrophilic spots. This will allow to find proper phase diagrams and to identify in which conditions what mechanisms act. The work reported here also encourages numerical studies to extend the results and shed light onto the underlying physics, in conditions which are not attainable in our experimental conditions. This kind of experiments could help to find therapeutics to relieve diseases like respiratory distress syndrome that appears in many premature newborns. 
This work was partly supported by the Spanish MINECO (Grant n. FIS2014-54101-P). JGC acknowledges a postdoctoral scholarship from UTA.

\section{References}

1. J. Aitken, Proc. Roy. Soc. Edinburgh (20), 94 (1893)

2. L. Rayleigh, Nature 86, 416 (1911)

3. T. Baker, Philosophical Magazine Series 6 44(262), 752 (1922)

4. D. Beysens, C.M. Knobler, Phys. Rev. Lett. 57(12), 1433 (1986)

5. D. Beysens, Comptes Rendus Physique 7(9-10), 1082 (2006)

6. R. Picknett, R. Bexon, Journal of Colloid and Interface Science 61(2), 336 (1977)

7. M. Sokuler, G. Auernhammer, C. Liu, E. Bonaccurso, H.J. Butt, Europhys. Lett. 89, $36004(2010)$

8. A. Steyer, P. Guenoun, D. Beysens, C.M. Knobler, Phys. Rev. A 44(12), 8271 (1991)

9. J. Guadarrama-Cetina, R. Narhe, D. Beysens, W. González-Viñas, Phys. Rev. E 89(1), $012402(2014)$

10. J. Guadarrama-Cetina, A. Mongruel, W. González-Viñas, D. Beysens, EPL (Europhysics Letters) 101(1), 16009 (2013)

11. J. Guadarrama-Cetina, A. Mongruel, W. González-Viñas, D. Beysens, EPL (Europhysics Letters) 110(5), $56002(2015)$

12. M.G. Medici, A. Mongruel, L. Royon, D. Beysens, Phys. Rev. E 90(6), 062403 (2014)

13. S. Anand, K. Rykaczewski, S. Subramanyam, D. Beysens, K. Varanasi, Soft Matter 11(1), 69 (2015)

14. J. Guadarrama-Cetina, W. González-Viñas, Phys. Rev. E 87(5), 054401 (2013)

15. J.L. Viovy, D. Beysens, C.M. Knobler, Phys. Rev. A 37, 4965 (1988)

16. T.M. Rogers, K.R. Elder, R.C. Desai, Phys. Rev. A 38, 5303 (1988)

17. D. Seo, J. Lee, C. Lee, Y. Nam, Scientific reports 6, 24276 (2016)

18. T.R. Nielsen, J. Rose, J. Kragh, Applied Thermal Engineering 29(2), 462 (2009)

19. Y. Zhao, Y. Luo, J. Zhu, J. Li, X. Gao, ACS Appl. Mater. Interfaces 7(22), 11719 (2015)

20. M. Srinivasarao, D. Collings, A. Philips, S. Patel, Science 292(5514), 79 (2001)

21. B. Majaron, S. Kimel, W. Verkruysse, G. Aguilar, K. Pope, L. Svaasand, E. Lavernia, J. Nelson, Lasers in surgery and medicine 28(5), 469 (2001)

22. K. Varanasi, M. Hsu, N. Bhate, W. Yang, T. Deng, Applied Physics Letters 95(9), 094101 (2009)

23. K.K. Varanasi, T. Deng, J.D. Smith, M. Hsu, N. Bhate, Applied Physics Letters 97(23), $234102(2010)$

24. J. Guadarrama-Cetina, A. Mongruel, M.G. Medici, E. Baquero, A. Parker, I. MilimoukMelnytchuk, W. González-Viñas, D. Beysens, Eur. Phys. J. E 37(11), 109 (2014)

25. R.D. Narhe, W. González-Viñas, D.A. Beysens, Appl. Surf. Sci. 256, 4930 (2010)

26. Movie corresponding to an experiment of type $1 \mathrm{~L}$ substrate, seen from above. It allows to see the behavior at mid time and length scales. The drop in the center is on the lecithin patch. HFOV is $3.31 \mathrm{~mm}$ and the movie starts at $700 \mathrm{~s}$ after starting the experiment.

27. Movie corresponding to an experiment of type 0L substrate, from where fig. 4a-e images are extracted, seen from above. It allows to see the behavior at small time and length scales. HFOV is $0.3 \mathrm{~mm}$ and the movie starts at $401 \mathrm{~s}$ after starting the experiment.

28. X. Song, J. Zhai, Y. Wang, L. Jiang, The Journal of Physical Chemistry B 109(9), 4048 (2005)

29. B.C. Bunker, R.W. CarPick, R.A. Assink, M.L. Thomas, M.G. Hankins, J.A. Voigt, D. Sipola, M.P. de Boer, G.L. Gulley, Langmuir 16(20), 7742 (2000)

30. Movie corresponding to an experiment of type nL substrate, seen from above. It allows to see the behavior at large time and length scales. The big drops are on lecithin patches. HFOV is $10.8 \mathrm{~mm}$ and the movie starts at $5124 \mathrm{~s}$ after starting the experiment.

31. N. Jeon, K. Finnie, K. Branshaw, R. Nuzzo, Langmuir 13(13), 3382 (1997)

32. X. Sun, V. Damle, A. Uppal, R. Linder, S. Chandrashekar, A. Mohan, K. Rykaczewski, Langmuir 31(51), 13743 (2015)

33. H. Zhao, D. Beysens, Langmuir 11, 627 (1995) 